\title{
Parathyroid carcinoma: A report of six cases with a brief review of the literature
}

\author{
JUN CAO $^{1 *}$, CAN CHEN $^{2 *}$, QING-LIANG WANG ${ }^{1}$, JIA-JIE XU $^{1}$ and MING-HUA GE ${ }^{1}$ \\ ${ }^{1}$ Department of Head and Neck Surgery, Zhejiang Province Cancer Hospital, Hangzhou, Zhejiang 310022; \\ ${ }^{2}$ Department of Hematology, The Affiliated Hangzhou First People's Hospital of Zhejiang Chinese Medical University, \\ Hangzhou, Zhejiang 310006, P.R. China
}

Received October 8, 2014; Accepted July 7, 2015

DOI: $10.3892 / 01.2015 .3774$

\begin{abstract}
Parathyroid carcinoma (PC) is an uncommon endocrine malignancy and constitutes a rare cause of hyperparathyroidism. The current study presents the clinical features, laboratory findings, sensitivity of imaging modalities, surgical treatment and the long-term outcome of six patients, who were diagnosed with PC and treated in the Department of Head and Neck Surgery, Zhejiang Province Cancer Hospital (Hangzhou, China) over 13 years (February 1999-January 2012). Pre-operative recognition and intraoperative identification of this rare endocrine malignancy is extremely important, but require a high index of clinical suspicion. The primary treatment is surgical en bloc resection of the tumor and any involved surrounding structures, and it is of great importance, as the prognosis depends on the initial surgery. Radiation therapy and chemotherapy showed no evidence of effectiveness on PC, although certain data show a decreased risk of localized disease recurrence with the addition of radiation therapy. The prognosis of PC is variable and post-operative parathyroid hormone levels that do not decrease often indicate a poor prognosis or presence of other metastases.
\end{abstract}

\section{Introduction}

Parathyroid carcinoma (PC) is an extremely rare endocrine malignancy, accounting for an estimated $0.005 \%$ of all cancers (1). PC was first described in 1904 by de Quevain (2) in a patient who presented with a non-functioning lesion with normal parathyroid function, and since then, studies on PC have been published successively in the literature worldwide. Although the majority of patients with PC present functioning

Correspondence to: Dr Ming-Hua Ge, Department of Head and Neck Surgery, Zhejiang Province Cancer Hospital, 38 Guangji Road, Gongshu, Hangzhou, Zhejiang 310022, P.R. China

E-mail: gemingh@163.com

${ }^{*}$ Contributed equally

Key words: parathyroid carcinoma, endocrine malignancy, hyperparathyroidism, diagnosis, surgical treatment lesions with hyperparathyroidism, including extremely high levels of parathyroid hormone (PTH) and serum calcium (3-5), it be difficult to arrive at a diagnosis of PC pre-operatively or even intraoperatively, for the reason that benign parathyroid tumors can also present with hyperparathyroidism and occasionally the PC is non-functioning (6). In addition, the pathological diagnosis of malignancy is challenging. Due to its low frequency, there is a lack of understanding with regard to this endocrine malignancy, and its diagnosis and treatment therefore present formidable challenges. The majority of the pathological features of parathyroid carcinoma are not specific, and the diagnosis may depend on demonstration of local invasion of adjacent structures, despite the absence of metastasis. A previous study reported that the diagnosis of PC is usually based on a combination of clinical, histological signs and radiological in a context of atypical presentation of PC (7). At present, en bloc surgical resection remains the only curative treatment, and a trend towards aggressive surgical management has improved outcomes (8). Furthermore, mortality is always associated with generalized metastases and severe hypercalcemia, but due to its low frequency, the mortality rate is unknown.

In the present study, the clinical presentation, laboratory and imaging findings, surgical treatment and prognosis of six cases of PC diagnosed in the Department of Pathology, Zhejiang Province Cancer Hospital (Hangzhou, Zhejiang, China) are presented, along with a brief review of literature.

\section{Cases report}

Patients. All cases of PC were diagnosed during a period of 13 years, between February 1999 and January 2012, in the Department of Head and Neck Surgery of Zhejiang Province Cancer Hospital (Hangzhou, China). None of the patients had a history of familiar hyperparathyroidism. In all cases, the diagnosis of PC was established by operative and pathological findings, combined with the clinical presentation, such as elevated serum calcium and PTH levels (Fig. 1). The experience gained in the management of this tumor is presented. All patients were followed up every 6 months (Table I).

Case 1. A 48-year-old female was admitted to the Department of Head and Neck Surgery in September 2008, with a diagnosis 
of nodular goiter and diabetes. The patient reported nausea, vomiting, polydipsia, polyuria and fatigue for at least 2 years and also had experienced trouble walking for the last year. Surgery for nephrolithiasis was performed 20 years previously, however, episodes of renal colic and hematuria occurred thereafter, which the patient did not seek treatment for. During this hospitalization period, laboratory tests found a normal blood sugar level, but a high serum level of calcium $(3 \mathrm{mmol} / \mathrm{l}$; normal range, 2-2.6 mmol/l). Ultrasound (US) scans revealed a multinodular goiter of the thyroid gland. During exploratory surgery of the neck, a large $3.5-\mathrm{cm}$ tumor of the left upper parathyroid gland was found, firmly adhered to the left lobe of the thyroid gland. An en bloc resection of the parathyroid mass with near-total thyroidectomy was performed. The histological diagnosis showed a PC that was $3.8 \mathrm{~cm}$ in maximum diameter, with capsular and vessel invasion. However, the serum calcium and PTH levels did not return to normal after the surgery. After 3 months, computed tomography (CT) of the neck revealed a $2.5-\mathrm{cm}$ mass in the suprasternal fossa, and the tumor was resected en bloc with the ipsilateral neck lymph nodes in a second procedure. The pathological examination revealed PC metastasis. Due to economic reasons, the patient terminated therapy and was discharged from hospital. According to the patient's relatives, the patient succumbed due to generalized metastases and severe debilitating hypercalcemia 2 years after the second surgery.

Case 2. A 72-year-old male who had been diagnosed with nodular goiter was admitted to the Department of Head and Neck Surgery of Zhejiang Province Cancer Hospital in August 2011, for surgical management. The patient reported dizziness and anxiety that had persisted for almost half a year. US demonstrated a multinodular goiter and a $3 \times 2.5-\mathrm{cm}$ mass in contact with the lower pole of the right lobe of the thyroid gland. The laboratory tests revealed that the serum calcium level was mildly elevated $(2.87 \mathrm{mmol} / \mathrm{l})$, as well as the plasma PTH level (155.9 ng/l; normal range, 15-65 ng/l). The patient also underwent a pre-operative FNA biopsy, but no evidence of $\mathrm{PC}$ was found. The patient underwent a palliative near-total right thyroidectomy with en bloc resection of the enlarged parathyroid mass and VI lymphadenectomy. The pathology revealed PC and thyroid tissue with nodular hyperplasia. Serum calcium and PTH levels returned to normal on the first post-operative day. The patient remains alive and free of disease 3 years after the surgery.

Case 3. A 44-year-old male with a thyroid nodule that had been physically apparent for a week presented to the Department of Head and Neck Surgery of Zhejiang Province Cancer Hospital in April 2010. Upon physical examination, a palpable irregular mass was discovered at the left upper pole of the thyroid gland. However, the patient exhibited normal serum calcium and PTH levels, and did not present with symptoms of hypercalcemia. An US scan revealed a mass in the middle of the left thyroid gland, which raised the suspicion of thyroid carcinoma. During surgery, a firm, $1 \mathrm{x} 1-\mathrm{cm}$ mass was found at the left upper pole of the thyroid gland. A near-total thyroidectomy with en bloc resection of the mass was performed. The margins were found to be negative for neoplasm and the central neck dissection demonstrated no positive lymph nodes. The pathology revealed low-grade PC, with small nests of

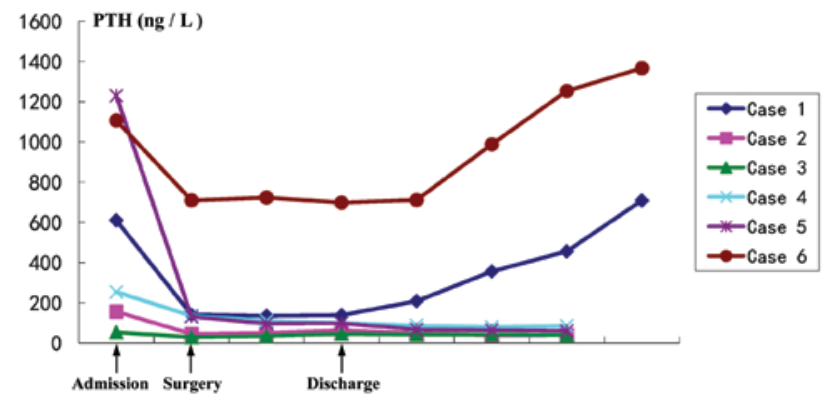

Figure 1. Parathyroid hormone (PTH) levels of 6 cases of pathyroid carcinoma. The normal range of PTH is $15-65 \mathrm{ng} / \mathrm{l}$.

carcinoma cells present in the surrounding thyroid tissue. The follow-up at 4 years revealed normal serum calcium and PTH levels. The patient has been free of recurrence since then.

Case 4. A 30-year-old female was admitted to the Department of General Surgery of Shaoxing People's Hospital (Shaoxing, China) in March 2005 and underwent en bloc resection of the left parathyroid with the diagnosis of primary hyperparathyroidism (PHPT). Following the surgery, the patient presented at the Department of General Surgery at Peking University Shenzen Hospital (Shenzen, China) in September 2011, with long-standing left-sided true vocal fold paralysis and subsequently underwent a workup by an otolaryngologist, which consisted of a thyroid US that demonstrated a left-sided thyroid nodule. The patient underwent a near-total thyroidectomy and the pathology indicated PC. However, the patient's hoarseness persisted and positron emission tomography-CT (PET-CT) imaging was performed, which showed recurrence of the right neck mass, within 4 months of the initial thyroidectomy. Calcium and PTH levels were beyond normal limits (serum calcium, $3.01 \mathrm{mmol} / 1$; and serum PTH, $254.1 \mathrm{ng} / \mathrm{l}$ ) at the time of the PET-CT. The patient was then referred to the Department of Head and Neck Surgery of Zhejiang Province Cancer Hospital in January 2012, for evaluation of the recurrent right neck mass. According to the previous examination, it was decided to excise the mass with a central neck dissection to achieve a pathological diagnosis and disease control. During surgery, a hard, $0.8-\mathrm{cm}$ mass was found near to the right recurrent laryngeal nerve. The tumor was resected en bloc with a number of the left cervical lymph nodes. The histological diagnosis showed no evidence of metastasis, but the serum calcium and PTH levels remained high after the surgery. At a follow-up at 2.5 years post-surgery, the patient was free of recurrence.

Case 5. A 69-year-old female was admitted to the Department of General Surgery of Zheijiang Province People's Hospital (Hangzhou, China) in November 2011. The patient underwent traditional high ligation and stripping of the great saphenous vein, for the presence of varicosis of 3 years previously. Following the surgery, the clinical symptoms improved. However, 2 months ago, the symptoms relapsed and the patient was admitted to the Department of Head and Neck Surgery of Zhejiang Province Cancer Hospital in February 1992, and on physical examination, a palpable irregular mass was found at the left lower pole of the thyroid 


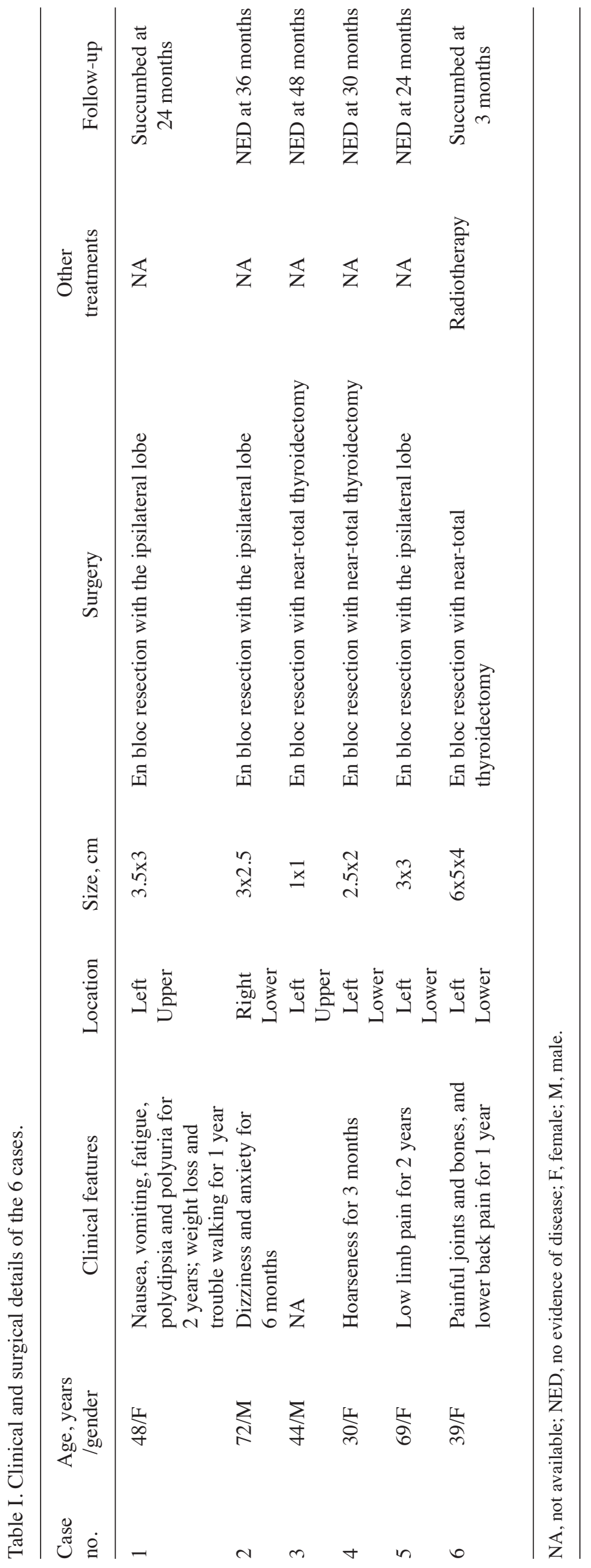



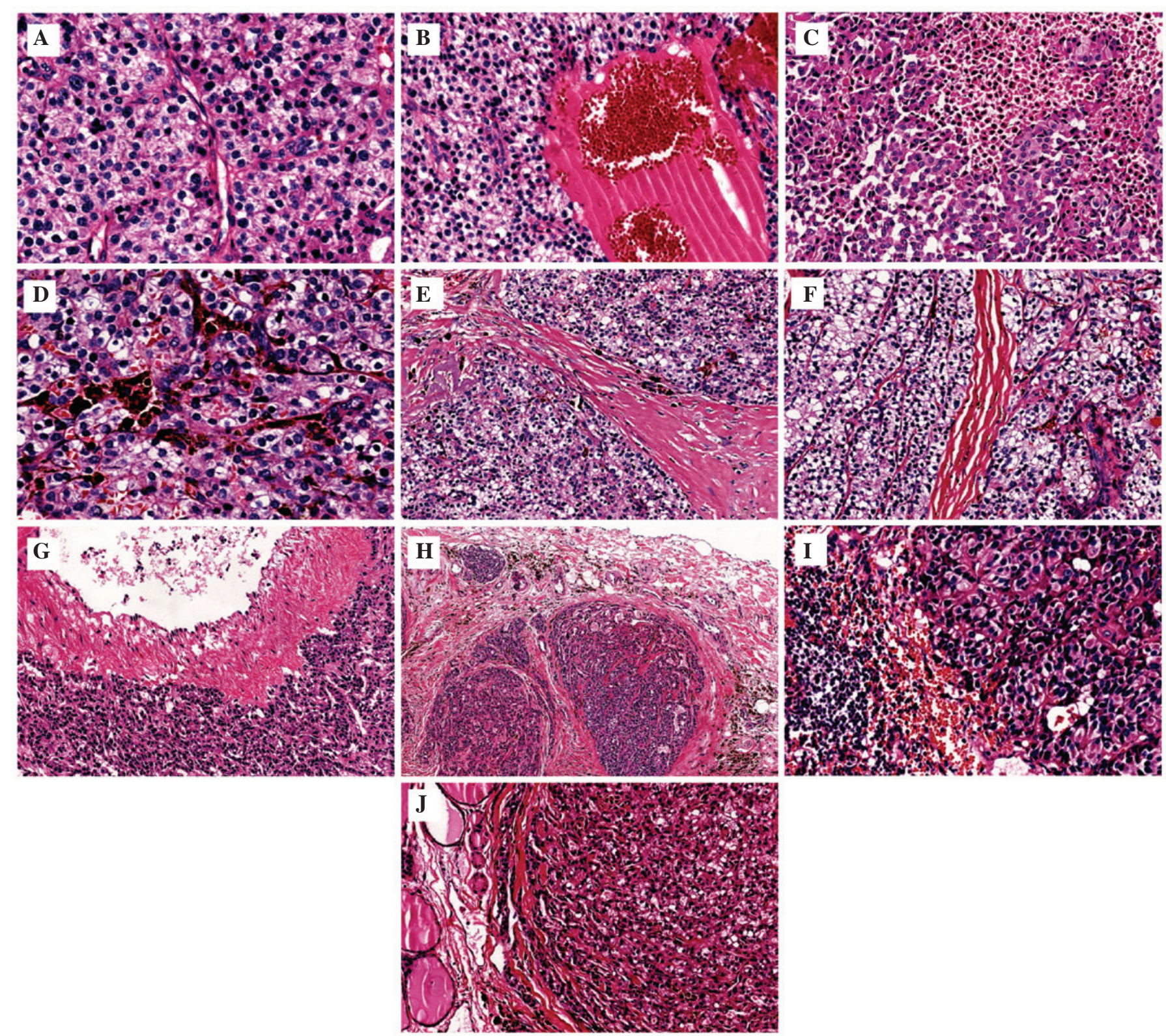

Figure 2. Hematoxylin and eosin (HE) staining of parathyroid carcinoma tissue. (A) Case 2, HE, x100 magnification. The nuclei of the tumor cells had an apparent unusual type, with clear cytoplasm. (B) Case 6, HE, x40 magnification. Cystic structure containing blood. (C) Case 2, HE, x40 magnification. Tumor necrosis. (D) Case 2, HE, x100 magnification. Pigmentation. (E) Case 4, HE, x40 magnification. Sheets or lobules of tumor cells separated by dense fibrous bands. (F) Case 6, HE, x40 magnification. Numerous blood vessels inside the tumor tissue. (G) Case 1, HE, x40 magnification. Infringement of the blood vessel walls. (H) Case 5, HE, x40 magnification. Capsular invasion. (I) Case 1, HE, x100 magnification. Lymphatic metastasis. (J) Case 3, HE, x40 magnification. Tumor cells invading the thyroid tissue.

gland. Laboratory tests established the diagnosis of PHPT. The patient's serum calcium and PTH levels were $3.44 \mathrm{mmol} / 1$ and $131.6 \mathrm{ng} / \mathrm{l}$, respectively, at the time of surgery. During the surgery, a hard $3 \times 3-\mathrm{cm}$ mass was discovered in the back of the left lobe of the thyroid gland. The mass was resected en bloc with the ipsilateral thyroid lobe. The calcium and PTH levels returned to normal immediately. The histological diagnosis showed PC with incipient capsular invasion. The patient is currently alive and free of disease 2 years after the surgery.

Case 6. A 39-year-old female patient with the diagnosis of thyroid carcinoma was transferred to the Department of Head and Neck Surgery of Zhejiang Province Cancer
Hospital in February 1999. The patient reported bone pain that had persisted for almost for a year, mostly in the lower extremities and the lower back. A month ago, these symptoms became worse, and the patient was unable to walk or even stand. X-ray scanning of the lower limbs revealed areas of osteolysis consistent with metastases. US revealed a $6 \times 5 \times 4-\mathrm{cm}$ mass in contact with the lower pole of the left lobe of the thyroid gland. Computed tomography scan of the thorax showed a solitary pulmonary nodule in the left lung consistent with metastasis. During surgery, the tumor behind the left lobe of the thyroid gland was resected en bloc with the ipsilateral thyroid lobe. The pathology revealed PC, and capsular invasion was apparent. Pre-operative calcium levels were elevated (2.7-3.3 mmol/l); however, normocalcemia 
was achieved immediately. The patient also underwent postoperative radiotherapy: The conventional fractionation of $1.8 \mathrm{~Gy}$ per fraction and 5 fractions/week for 5 weeks (total dose, 45 gy). However, 3 months later, the patient's clinical condition deteriorated due to generalized metastases and the patient succumbed to cachexia.

\section{Discussion}

PC has, until recently, been regarded as an exceedingly rare malignancy that is mostly observed in patients treated for PHPT or thyroid tumors. The tumors occur equally in men and women, in contrast to benign PHPT, which affects more women than men. PC is a slow-growing tumor of low malignant potential, but is a serious and frustrating clinical problem. $\mathrm{PC}$ is generally associated with high calcium and extremely high PTH levels. Patients are more likely to be symptomatic at the time of presentation, and usually present with the symptoms of excessive PTH excretion, including renal colic, painful joints and bones or lower back pain, nocturia, thirst, polydipsia, anorexia, gastrointestinal disturbances, abdominal pain, weight loss and neuromuscular complaints, such as weakness and fatigue (9). The majority of these tumors are functioning, however, they can also occur as a non-functioning $\mathrm{PC}$. The current study presents an even rarer case of PC with normal serum calcium and PTH levels, no symptoms of hypercalcemia, and PC identified during thyroidectomy. Few cases of incidental non-functioning PC have been described in the last 100 years (20 cases since 1929) (10). Non-functioning PC is usually clinically silent until it presents with signs and symptoms of local growth and invasion (e.g., hoarseness, a neck mass and dysphagia).

No single laboratory finding is diagnostic of PC; similar to benign PHPT, levels of serum calcium and PTH are elevated in functioning PC. However, hypercalcemia in PC is usually more profound, with a serum calcium level that is $>3.0 \mathrm{mmol} / 1$ and PTH levels as much as 1,230 ng/l above the upper limit of normal (Fig. 1). These levels are mildly elevated or near the upper limit of normal in the majority of benign PHPT cases. In a study by Schaapveld et al (11), it was demonstrated that a PTH level 10 times the upper limit of the normal range had a positive predictive value of $81 \%$ for PC. Furthermore, as shown in Fig. 1 of the present study, a post-operative PTH level that did not decrease often indicated a poor prognosis or the presence of other metastases. In addition, serum levels of alkaline phosphatase, hypophsphatemia and hyperchloremic metabolic acidosis are also raised in certain patients with PC (12). Recently, elevated plasma human chorionic gonadotropin levels were documented in certain patients with PC, but were normal in benign disease (13).

The commonly used imaging modalities include US, CT, magnetic resonance imaging (MRI), PET and sestamibi scans (14-17). These imaging studies are not very effective in determining the size and location of abnormal parathyroid glands. However, the sensitivity and specificity of these imaging modalities are increased when they are used in combination. Ultrasonography is the most widely used tool to localize primary and locally recurrent disease. Typical features of PCs are lobulated, hypoechoic and relatively larger parathyroid glands with ill-defined borders compared with adenomas (14). Reeder et al (15) found that US has $100 \%$ accuracy in detecting parathyroid lesions of $\geq 1 \mathrm{~cm}$. In the present study cases, US revealed a parathyroid neoplasm in one case only, where the size of the neoplasm was $6 \mathrm{~cm}$, and probably due to the coexistence of multinodular goiter in the remaining 5 cases, as it was also proven intraoperatively. Similar to US, sestamibi scanning has gained wide acceptance as a precise method of assessing parathyroid lesions, and it was performed in 3 of these cases (cases 1,4 and 6), demonstrating the localization of the parathyroid adenomas. However, sestamibi scans provide little benefit in differentiating benign from malignant parathyroid lesions (16). Other diagnostic techniques, such as CT, MRI and PET, may be useful when other imaging modalities fail to localize the parathyroid neoplasm, and they are essential in the identification of metastases and recurrent disease once the diagnosis has been made (17).

The pre-operative diagnosis of PC can be extremely challenging. In the present study, no patient was diagnosed with PC prior to surgery (4 patients were suspected to have thyroid tumors and 2 were suspected to have parathyroid adenomas). The diagnosis becomes evident only after cytological and/ or histological analysis. However, fine-needle aspiration (FNA) biopsy should be avoided when PC is suspected. Firstly, it is difficult to differentiate between benign and malignant parathyroid lesions on FNA biopsy (18). Secondly, danger of possible procedure-related complications, such as bleeding and tumor seeding along the needle tract has been reported (19). Only 1 patient (case 2) underwent pre-operative FNA biopsy in the present study, but no evidence of PC was found. Final histopathological descriptions of $\mathrm{PC}$ reported in the 6 cases were varied and included capsular and vascular invasion, sheets or lobules of tumor cells separated by dense fibrous bands, pigmentation, necrosis and hemorrhage (Fig. 2). However, these pathological features are not always present in PC. Furthermore, a number of these features may also be observed in parathyroid adenomas or thyroid tumors. Therefore, just like for other endocrine malignancies, the diagnosis of PC is also based on the clinical presentation.

PCs are usually treated surgically, and the main treatment goal should be the total removal of the PC with negative margins. The majority of studies recommend en bloc resection at the time of the initial surgery to offer the best opportunity for a cure. Therefore, a high index of pre-operative suspicion and the recognition of the possibility of malignancy during exploration are extremely important. Adequate surgical excision includes en bloc resection of the tumor together with the ipsilateral thyroid lobe, and excision of any adjacent structures involved (9). The surgery must be performed carefully in order to avoid rupturing the capsule of the tumor and seeding of the surgical field, and the recurrent laryngeal nerve should be resected only when it is involved and not functioning. Prophylactic or radical neck resection is usually performed only if enlarged or abnormal-appearing lymph nodes are found to be involved, as unnecessary prophylactic radical neck dissection may increase the risk of surgical complications (20). In the present cases, en bloc resection of the tumor with near-total thyroidectomy was performed. In cases 1,5 and 6 , the ipsilateral thyroid lobe was resected en bloc due to local infiltration, and in cases 
2, 3 and 4, this was due to multinodular goiters. The most common relapse pattern after the initial surgery is local recurrence and distant metastases (e.g., lung, bone and liver metastases). When technically possible, the resection of local recurrence and metastatic lesions is recommended. Although an absolute curative treatment is unlikely, this measure is justified for the reduction of severe hypercalcemia, which is the main lethal factor of advanced metastatic PC (21). Until recently, the data have been limited for the treatment of PC with radiation therapy and chemotherapy. PC has been traditionally considered as a radio-resistant malignancy, with mainly disappointing results observed in the majority of patients who are administered radiation therapy. However, a recent retrospective study has advocated that post-operative radiation therapy may be a strategy for optimizing local control, which may produce a positive benefit on survival (1). In case 6 of the present study, radiation therapy and chemotherapy were attempted as treatment for the bone and lung metastases, but it was not shown to be effective in improving the survival or correcting the hypercalcemia of this patient.

The prognosis of PC is variable. Disease recurrence occurs in at least $50 \%$ of cases, and is usually indicated by elevated PTH and serum calcium levels upon re-examination. In a previously published study, the overall 5- and 10-year survival rates ranged between $<50$ and $85 \%$, and between 35 and $79 \%$, respectively. Early identification, appropriate initial surgery, medical treatment for hypercalcemia and selective use of radiation therapy offers the best prognosis (22). In the present cases, only two patients developed recurrence within 2 years and succumbed due to generalized metastases and severe debilitating hypercalcemia. The remaining patients are alive and remain free of disease after their first surgeries.

To conclude, the present case study highlighted that more attention should be focused on patients with high levels of PTH and serum calcium, and if there is a mass near the thyroid gland, PC should be highly suspected. Furthermore, the information gained may assist in the identification and treatment of the disease in a more timely and adequate manner. Due to their rarity, a multi-institutional pooling of cases is required for more insight into $\mathrm{PC}$ in order to further understand this disease and to develop more effective therapeutic methods.

\section{Acknowledgements}

This study was supported by the National Natural Science Foundation of China (no. 81202127), the Natural Science Foundation of Zhejiang Province (no. LY14H160014) and the Traditional Chinese Medicine Science and Technology Plan of Zhejiang Province (no. 2013ZA025).

\section{References}

1. Wei $\mathrm{CH}$ and Harari A: Parathyroid carcinoma: Update and guidelines for management. Curr Treat Options Oncol 13: 11-23, 2012.

2. De Quevain F: Malignant aberrant parathyroid. Dtsch Z Fuer Chir 100: 334-352, 1904. (In German).

3. Cryns VL, Rubio MP, Thor AD, Louis DN and Arnold A: p53 abnormalities in human parathyroid carcinoma. J Clin Endocrinol Metab 78: 1320-1324, 1994.

4. Meckeown PP, Mcgarity WC and Sewell CW: Carcinoma of the parathyroid gland: Is it overdiagnosed? A report of three cases, Am J Surg 147: 292-298, 1984.

5. Sandelin K, Auer G, Bondeson L. Grimelius L and Farnebo LO: Prognostic factors in parathyroid cancer: A review of 95 cases, World J Surg 16: 724-731, 1992.

6. Levin KE, Galante M and Clark $\mathrm{OH}$ : Parathyroid carcinoma versus parathyroid adenoma in patients with profound hypercalcaemia. Surgery 101: 649-660, 1987.

7. Rodriguez C, Naderi S, Hans C and Badoual C: Parathyroid carcinoma: A difficult histological diagnosis. Eur Annals Otorhinolaryngol Head Neck Dis 129: 157-159, 2012.

8. Kassahun WT and Jonas S: Focus on parathyroid carcinoma. Int J Surg 9: 13-19, 2011.

9. Abdelgadir Adam M, Untch BR and Olson JA Jr: Parathyroid carcinoma: Current understanding and new insights into gene expression and intraoperative parathyroid hormone kinetics. Oncologist 15: 61-72, 2010.

10. Mazeh H, Prus D and Freund HR: Incidental non-functional parathyroid carcinoma identified during thyroidectomy. Isr Med Assoc J 10: 659, 2008.

11. Schaapveld M, Jorna FH, Aben KK, Haak HR, Plukker JT and Links TP: Incidence and prognosis of parathyroid gland carcinoma: A population-based study in The Netherlands estimating the preoperative diagnosis. Am J Surg 202: 590-597, 2011.

12. Haghighi P, Astarita RW, Wepsic HT and Wolf PL: Concurrent primary parathyroid hyperplasia and parathyroid carcinoma. Arch Pathol Lab Med 107: 349-350, 1983.

13. Rubin MR, Bilezikian JP, Birken S and Silverberg SJ: Human chorionic gonadotropin measurements in parathyroid carcinoma. Eur J Endocrinol 159: 469-474, 2008

14. Tamler R, Lewis MS, LiVolsi VA and Genden EM: Parathyroid carcinoma: Ultrasonographic and histologic features. Thyroid 15: 744-745, 2005.

15. Reeder SB, Desser TS, Weigel RJ and Jeffrey RB: Sonography in primary hyperparathyroidism: Review with emphasis on scanning technique. J Ultrasound Med 21: 539-552, 2002.

16. Al-Sobhi S, Ashari LH and Ingemansson S: Detection of metastatic parathyroid carcinoma with Tc-99 m sestamibi imaging. Clin Nucl Med 24: 21-23, 1999.

17. Sandelin K: Parathyroid carcinoma. Cancer Treat Res 89: 183-192, 1997.

18. Mohebati A, Shaha A and Shah J: Parathyroid carcinoma: Challenges in diagnosis and treatment. Hematol Oncol Clin North Am 26: 1221-1238, 2012.

19. Spinelli C, Bonadio AG, Berti P, Materazzi G and Miccoli P: Cutaneous spreading of parathyroid carcinoma after fine needle aspiration cytology. J Endocrinol Invest 23: 255-257, 2000.

20. Clayman GL, Gonzalez HE, El-Naggar A and Vassilopoulou-Sellin R: Parathyroid carcinoma: Evaluation and interdisciplinary management. Cancer 100: 900-905, 2004.

21. Givi B and Shah JP: Parathyroid carcinoma. Clin Oncol (R Coll Radiol) 22: 498-507, 2010.

22. Harari A, Waring A, Fernandez-Ranvier G, Hwang J, Suh I, Mitmaker E, Shen W, Gosnell J, Duh QY and Clark O: Parathyroid carcinoma: A 43-year outcome and survival analysis. J Clin Endocrinol Metab 96: 3679-3686, 2011. 